\title{
Visualization of large microarray experiments with space maps
}

\section{Nils Gehlenborg*1,2 and Alvis Brazma ${ }^{1}$}

\author{
Address: ${ }^{1}$ European Bioinformatics Institute, Cambridge, CB10 1SD, UK and ${ }^{2}$ Graduate School of Life Sciences, University of Cambridge, \\ Cambridge, CB2 1RX, UK \\ E-mail: Nils Gehlenborg* - nils@ebi.ac.uk \\ * Corresponding author
}

from Fifth International Society for Computational Biology (ISCB) Student Council Symposium

Stockholm, Sweden 27 June 2009

Published: 19 October 2009

BMC Bioinformatics 2009, I0(Suppl 13):O7 doi: 10.1186/147I-2105-10-S13-O7

This article is available from: http://www.biomedcentral.com//47I-2/05//0/SI3/O7

(C) 2009 Gehlenborg and Brazma; licensee BioMed Central Ltd.

This is an open access article distributed under the terms of the Creative Commons Attribution License ( which permits unrestricted use, distribution, and reproduction in any medium, provided the original work is properly cited.

\section{Background}

Heatmaps and profile plots are effective techniques to visualize expression profiles of several hundred genes across a few dozen samples. However, these techniques do not scale to data sets with expression profiles that have been measured across several hundred samples or even thousands of samples. Our motivation to find a solution to this scaling problem is based on the observation that with increasingly mature and affordable microarray platforms, the number of studies in ArrayExpress [1] including hundreds of samples has been increasing steadily over the years.

\section{Methods}

We have developed the glyph-based Space Maps visualization technique that is conceptually similar to Value and Relation Displays [2]. The technique comprises two steps: (1) Generation of glyphs to represent gene expression profiles and (2) arrangement of the glyphs to reflect relationships between genes. Both steps support the integration of biological knowledge into the visualization, for instance in form of ontologies that describe hierarchical relationships among the conditions in the data. We also use hierarchical organization of samples and aggregation of expression levels to summarize expression values of groups of samples, which enables the user to reduce the amount of data shown on each glyph. Similar to treemaps [3], this construction makes it possible to start out with an overview of the data and then view details on demand.

\section{Results}

We have applied the Space Maps visualization to a data set with 5,372 samples (Margus Lukk, personal communication). This data set has been constructed from a large collection of publicly available gene expression data sets and a problem-specific hierarchy on the samples is available. We selected the 1,000 most variable genes from this data set and visualized this subset with our technique (Figure 1). The arrangement of the glyphs represents an overview of the global patterns in the data, such as clusters and outliers. Furthermore, the visualization provides insight into local patterns in the gene expression profiles. Since global patterns arise directly from local patterns we were able to explain several of the clusters and outliers and assign meaningful labels to them.

\section{Conclusion}

The Space Maps visualization technique is a novel approach to visualization of gene expression data that facilitates the visualization of expression profiles of genes with hundreds or thousands of samples without loss of context information. A major strength of this technique is that it allows a tightly coupled exploration of local and 

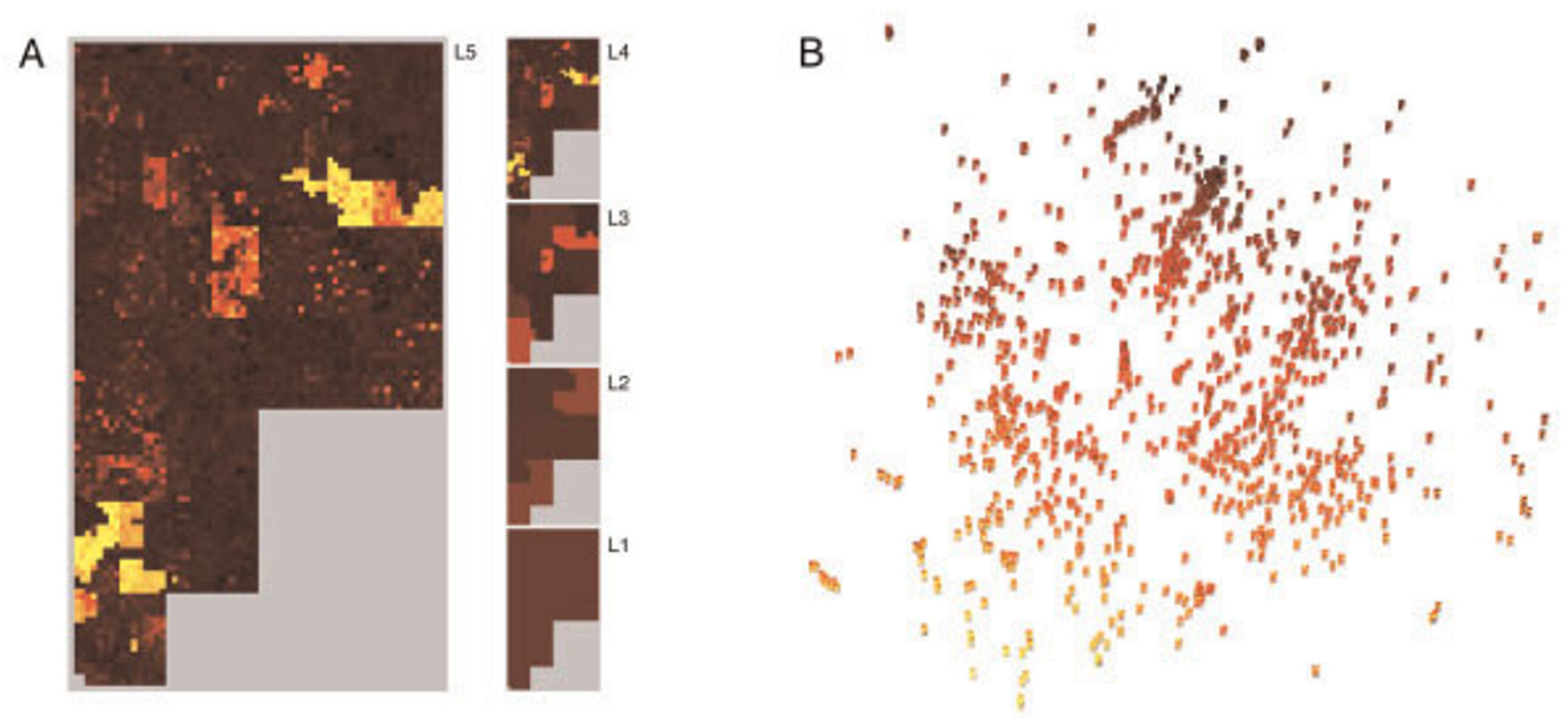

Figure I

Space Maps visualization of I,000 genes with 5,372 samples. (A) An expression profile at five levels of the hierarchy. Level LI corresponds to the root and Level L5 corresponds to the leafs of the hierarchy. The information-content of the glyph increases as the levels increase. (B) A non-linear projection [4] of I,000 expression profiles into 2D space. It is possible to make out global patterns such as clusters and outliers. Local patterns in the expression profiles can be identified as well, for instance in the lower left corner.

global patterns, which makes hypothesis generation more efficient than with traditional techniques.

\section{References}

I. Parkinson $\mathrm{H}$, et al: ArrayExpress update - from an archive of functional genomics experiments to the atlas of gene expression. Nucleic Acids Res 2007, 37(Database issue): D868-D872.

2. Yang J, et al: Value and Relation Display - Interactive visual exploration of large data sets with hundreds of dimensions. IEEE Transactions on Visualization and Computer Graphics 2007, I3:494-507.

3. Johnson B and Shneiderman B: Tree maps: A space-filling approach to the visualization of hierarchical information structures. Proceedings of the 2nd International IEEE Visualization Conference 199|, 284-29|.

4. Venna J and Kaski S: Non-linear dimensionality reduction as information retrieval. Proceedings of the II th International Conference on Artificial Intelligence and Statistics (AISTATS 2007) 2007, 568-575.
Publish with BioMed Central and every scientist can read your work free of charge

"BioMed Central will be the most significant development for disseminating the results of biomedical research in our lifetime. "

Sir Paul Nurse, Cancer Research UK

Your research papers will be:

- available free of charge to the entire biomedical community

- peer reviewed and published immediately upon acceptance

- cited in PubMed and archived on PubMed Central

- yours - you keep the copyright

Submit your manuscript here:

http://www.biomedcentral.com/info/publishing_adv.asp
BioMedcentral 